\title{
CRISIS DEL PETRÓLEO Y POLÍTICA ENERGÉTICA
}

\begin{abstract}
RESUMEN
Se espera un elevado crecimiento de la producción en los países desarrollados, caracterizado por un alto consumo de energía cuya tendencia es también creciente. En este escenario, una política energética nacional (que reduzca la vulnerabilidad de nuestra economía, permita un ahorro en el consumo de energía, y fomente la producción y diversificación tecnológica) debe considerar la seguridad energética como prioridad para garantizar un desarrollo sostenible en el futuro y para alcanzar el desafío de la autosuficiencia energética; la conservación del medio ambiente, a través de la promoción y desarrollo de proyectos de energías renovables; invertir en eficiencia energética; y por último, la modernización y fortalecimiento de Petroperú. Estas políticas implica un cambio de enfoque desde el corto al mediano y largo plazo en el manejo de nuestros recursos.
\end{abstract}

Palabras Clave: Energía, política energética, desarrollo sostenible

\begin{abstract}
It is expected a growth in the production production high growth in developed countries distinguished by high energy consumption and this trend is also increasingly. In this scenary, a national energy policy which reduces vulnerabilities in our economy, permits to do savings in energy consumption and promote production and technological diversification, must consider energy security as main priority in order to guarantee a sustained development in the future and to reach the challenge of an energy autosufficiency; enviromental conservation through the promotion and development of renewable energy projects; investment in energetic efficiency and finally the modernization and strengthen of Petroperú. These policies imply a focus change from short term to the medium and long term in the management of our resources.
\end{abstract}

Key Words: Oil, energetic policy, sustainable development.

* Doctor en Ciencias Económicas. Profesor de la Facultad de Ciencias Administrativas (UNMSM). 
La economía mundial sigue inmersa en una de las fases cíclicas más favorables de las últimas décadas, y como revela el último informe de perspectivas del Fondo Monetario Internacional (FMI), se espera que el Producto Bruto Interno (PBI) mundial sea, a fines del año 2006, un $4.9 \%$ más alto que en 2005. Este auge económico podría incluso continuar en el 2007, ejercicio para el cual se anticipa un $4.7 \%$ de crecimiento. En consecuencia, se estaría encadenando un periodo de crecimiento económico muy duradero e intenso, gracias a una lenta desaceleración vigente desde 2004 (cuando se creció al 5.3\%).

Este mayor crecimiento económico se viene produciendo, principalmente, en aquellos países de elevado consumo de energía por unidad del PBI, como es el caso de Estados Unidos, cuyo crecimiento es del 3.5\%; China, cuyo ritmo es del $10 \%$, y la India, con $7.5 \%$. El país asiático con más dinamismo económico sigue siendo China, que en los últimos veinticinco años ha tenido un crecimiento promedio del 9\% anual, alcanzando el segundo mayor PBI del mundo y, como todo parece indicar, su expansión no se detendrá al menos durante los próximos diez años.

Según las proyecciones de la Agencia Internacional de la Energía (IEA) ${ }^{1}$, al 2030 la demanda de energía se incrementará en el mundo al ritmo de un $2.1 \%$ anual, porcentaje superior al de los últimos veinte años. En valores absolutos, el consumo diario será de 121,3 millones de barriles, un $12.1 \%$ para China y un $5.6 \%$ para India. Actualmente el consumo total es de 84 millones de barriles por día y un $7 \%$ corresponde al gigante asiático. La tendencia del consumo energético de China, India y otros países refleja que en un fu-

Gráfico $1^{2}$

Evolución del precio del petróleo (2003-julio 2006)

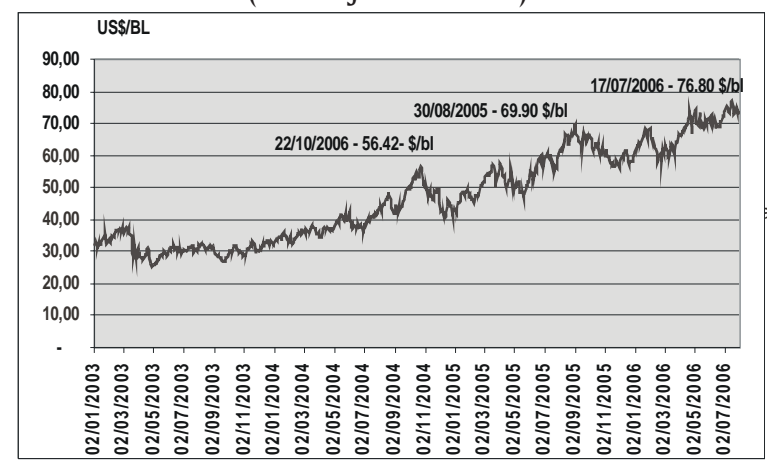

Fuente: Bloomberg, 2006 y elaboración propia. turo próximo la demanda de petróleo superará la oferta disponible por encima de los cien dólares el barril (véase Gráfico 1).

\section{LOS PILARES DE LA POLÍTICA ENERGÉTICA}

En este escenario, reflexiones sobre las tendencias de los mercados energéticos, la preocupación por la planificación a mediano y largo plazo, los problemas de vulnerabilidad de nuestra economía al suministro energético, el ahorro en el uso de este factor de producción ${ }^{3}$, la discusión sobre las tecnología disponibles en materia de diversificación energética, la política de precios coherente con los objetivos de mediano y largo plazo, etc., son temas relevantes en la política energética del gobierno. Por lo tanto, el debate debería girar en torno a los siguientes pilares:

1. La seguridad energética como prioridad principal para garantizar un desarrollo sostenible en el futuro. La garantía de un suministro energético estable para nuestras generaciones presentes y futuras es una condición prioritaria y necesaria para asegurar el suministro de todos los bienes y servicios, por cuanto la energía es un input fundamental de todo proceso productivo. El precio de los servicios energéticos repercute en el precio de aquellos bienes y servicios, por lo tanto, el control de esta variable resulta clave para la competitividad económica de las empresas y para evitar tensiones inflacionarias que limiten el crecimiento y desarrollo económico del país.

Desde 1990, nuestra Balanza Comercial de Hidrocarburos es deficitaria (véase Gráfico 2), llegando aproximadamente a 508 millones

\section{Gráfico 2}

Balanza Comercial de Hidrocarburos

(1990-2005)

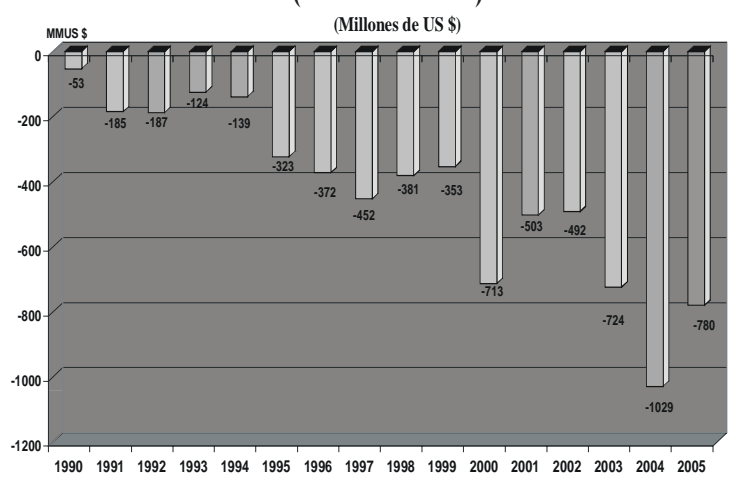

Fuente: Ministerio de Energia y Minas-Dirección General de Hidrocarburos y PERUPETRO, 2005 y elaboración propia. 
de dólares entre enero y mayo del 2006. Las reservas de petróleo, al ritmo de la producción actual, sólo durarían catorce años más (véase Gráfico 3).

El desafío es revertir esta tendencia y alcanzar la autosuficiencia energética, para lo cual existen dos vías: una es persistir en la pro-

Gráfico 3

Reservas probadas de petróleo crudo (1990-2005)

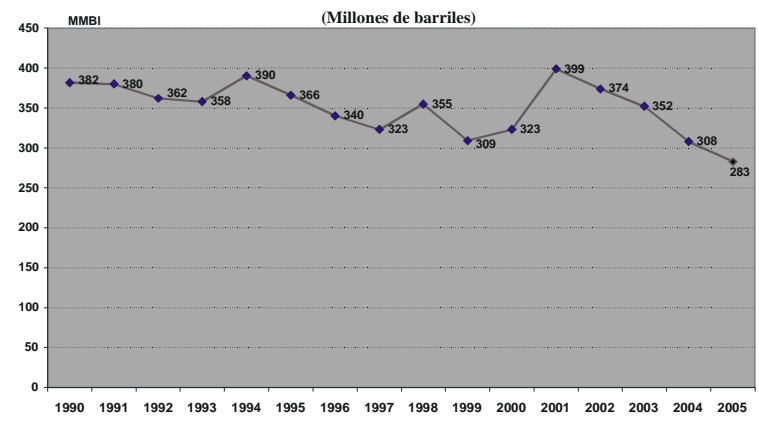

Fuente: Ministerio de Energía y Minas y Plan Referencial de Hidrocarburos 2003 - 2012 y elaboración propia

moción de las inversiones en el sector, exigiendo al mismo tiempo más eficiencia a la administración de los contratos de licencia de exploración y explotación firmados por Perupetro con las empresas privadas. La otra vía es diseñar estrategias de promoción y desarrollo de fuentes de energía alternativas a las tradicionales (solar, eólica, hidráulica, biocarburantes, etc.), con el apoyo del Estado. Debemos apuntar hacia un Plan Nacional de Energías Renovables que incorpore incentivos fiscales a iniciativas empresariales, así como a proyectos de Investigación y Desarrollo (I+D).

2. Conservación del medio ambiente. El Perú es un país con un nivel de emisiones considerado alto, con déficit tecnológico, y especialmente sensible a los efectos del cambio climático, tanto en términos estrictamente ambientales como en términos económicos y sociales (recordemos los daños causados por el Fenómeno de El Niño y las sequías en la zona sierra del país). Entre los instrumentos que el Protocolo de Kyoto contempla para que los países firmantes cumplan con sus objetivos de reducción de emisiones y para que generen un desarrollo sostenible, figuran los llamados Mecanismos de Desarrollo Limpio (MDL) que pueden ser aprovechados por nuestro país, como ya lo vienen haciendo, China, India y Brasil. La promoción y el desarrollo de proyectos de energías renovables apuntaría en la dirección de los MDL por un lado y, por otro, ayudaría a encarar el reto energético por las posibilidades que el futuro aguarda a este tipo de energías necesarias para nuestra calidad de vida. Actualmente, estas energías constituyen el sector de más auge de la industria energética, como se viene dando en la Unión Europea, donde la energía eólica y la fotovoltaica tienen un índice de crecimiento superior al $20 \%$ anual. Cabe resaltar que el desarrollo del conjunto de las energías renovables ha creado más de $300 \mathrm{mil}$ puestos de trabajo.

3. Invertir en eficiencia energética. Los países y sus economías son más competitivos en la medida que aumenta su eficiencia energética, es decir, en la medida que el consumo de energía por unidad de producto producido o de servicio prestado sea cada vez menor. La tecnología disponible, por un lado, y los hábitos de consumo responsables, por el otro, hacen posible y rentable consumir menos energía mejorando la competitividad de las empresas y la calidad de vida personal. Incentivar inversiones en investigación, desarrollo e innovación $(\mathrm{I}+\mathrm{D}+\mathrm{I})$ para hacer los procesos productivos menos intensivos en el uso de la energía; actualizar normas que rigen el sector de la construcción adaptándolas a las nuevas necesidades de energía y ambientales, diseñar Planes de Ahorro y Eficiencia Energética con un conjunto de medidas y objetivos, etc., son algunos temas que debería incorporar la política energética del país para optimizar el uso de energía.

4. Modernización y fortalecimiento de Petroperú. La tendencia mundial de las empresas petroleras estatales es hacia el fortalecimiento y expansión; en cambio, en el Perú ocurre lo contrario. Prueba de ello es la indefinición que actualmente existe respecto al futuro de Petroperú, lo cual considero personalmente un grave error de cara al futuro. Recordemos que esta empresa aporta al tesoro público aproximadamente 4,000 millones de soles anuales y juega el papel de regulador en el mercado de combustibles, evitando abusos de dominio. Estas son razones más que suficientes para que el próximo gobierno asuma el reto de seguir el ejemplo de Petrobrás, Petroecuador, Ecopetrol, ENAP ${ }^{4}$, etc. 


\section{A MODO DE CONCLUSIÓN}

Si como todo parece indicar, estamos entrando a una época de precios del petróleo relativamente elevados con consecuencias negativas sobre la productividad, al tiempo que aumenta las exigencias en materia medioambiental (el Protocolo de Kyoto debe considerarse tan sólo el primer paso en un proceso histórico cada vez más poderoso de aumento de relevancia de los criterios medioambientales en éste y en otros campos), creo que es de rigor señalar aquí que nuestra política energética requiere un rápido cambio de enfoque hacia consideraciones de mediano y largo plazo, y ello exige aplicar el concepto horizontal de desarrollo sostenible basado en los siguientes pilares: la seguridad en el suministro, la eficiencia y calidad de dicho suministro, y el respeto al medio ambiente.

\section{NOTAS}

1 La Agencia Internacional de Energía (IEA) es un organismo autónomo establecido tras la crisis del petróleo de 1973, en noviembre de 1974, dentro del marco de la OCDE para implementar un programa de energía a nivel internacional.
2 Véase Informes de la Agencia Internacional de Energía (20052006).

3 El control de la energía es clave, de su escasez dependen los precios de casi todos los bienes en el mercado. Los alimentos son más caros si el petróleo sube al igual que el transporte en todas sus formas la industria y la gama de actividades que derivan de ellas.

4 Un desarrollo más específico sobre los pilares de una nueva política energética puede verse en Narváez Liceras, A. (2006). Diario La República, 15 de julio del 2006.

\section{BIBLIOGRAFÍA}

Agencia Internacional de Energía (2005).

Ministerio de Energía y Minas - Dirección General de Hidrocarburos (2006).

Ministerio de Energía y Minas - Dirección General de Hidrocarburos (2002). Plan Referencial de Hidrocarburos 2003-2012.

Narváez Liceras, Alejandro (2006). «Para un debate sobre política energética». Diario $L a$ República, 10 de julio del 2006. 